\title{
Clinical symptoms of androgen deficiency in men with migraine or cluster headache: a cross-sectional cohort study
}

Iris E. Verhagen ${ }^{1,2 \dagger} \mathbb{D}$, Roemer B. Brandt ${ }^{1 \dagger}$, Carlijn M. A. Kruitbosch ${ }^{1}$, Antoinette MaassenVanDenBrink², Rolf Fronczek ${ }^{1+}$ and Gisela M. Terwindt ${ }^{1 *+}$

\begin{abstract}
Background: To compare symptoms of clinical androgen deficiency between men with migraine, men with cluster headache and non-headache male controls.

Methods: We performed a cross-sectional study using two validated questionnaires to assess symptoms of androgen deficiency in males with migraine, cluster headache, and non-headache controls. Primary outcome was the mean difference in androgen deficiency scores. Generalized linear models were used adjusting for age, BMI, smoking and lifetime depression. As secondary outcome we assessed the percentage of patients reporting to score below average on four sexual symptoms (beard growth, morning erections, libido and sexual potency) as these items were previously shown to more specifically differentiate androgen deficiency symptoms from (comorbid) anxiety and depression.

Results: The questionnaires were completed by $n=534 / 853$ (63\%) men with migraine, $n=437 / 694$ (63\%) men with cluster headache and $n=152 / 209$ (73\%) controls. Responders were older compared to non-responders and less likely to suffer from lifetime depression.

Patients reported more severe symptoms of clinical androgen deficiency compared with controls, with higher AMS scores (Aging Males Symptoms; mean difference \pm SE: migraine $5.44 \pm 0.90, p<0.001$; cluster headache $5.62 \pm 0.99$, $p<0.001$ ) and lower qADAM scores (quantitative Androgen Deficiency in the Aging Male; migraine: $-3.16 \pm 0.50$, $p<0.001$; cluster headache: $-5.25 \pm 0.56, p<0.001$ ). Additionally, both patient groups more often reported to suffer from any of the specific sexual symptoms compared to controls (18.4\% migraine, $20.6 \%$ cluster headache, $7.2 \%$ controls, $p=0.001$ ).
\end{abstract}

Conclusion: Men with migraine and cluster headache more often suffer from symptoms consistent with clinical androgen deficiency than males without a primary headache disorder.

Keywords: Headache, Morning erections, Libido, Sexual potency, Sex hormones

\footnotetext{
* Correspondence:

${ }^{\dagger}$ Iris E. Verhagen and Roemer B. Brandt shared first authorship.

${ }^{\dagger}$ Rolf Fronczek and Gisela M. Terwindt shared last authorship.

'Department of Neurology, Leiden University Medical Center, P.O. 9600,

2300, WB, Leiden, the Netherlands

Full list of author information is available at the end of the article
}

(c) The Author(s). 2021, corrected publication 2021. Open Access This article is licensed under a Creative Commons Attribution 4.0 International License, which permits use, sharing, adaptation, distribution and reproduction in any medium or format, as long as you give appropriate credit to the original author(s) and the source, provide a link to the Creative Commons licence, and indicate if changes were made. The images or other third party material in this article are included in the article's Creative Commons licence, unless indicated otherwise in a credit line to the material. If material is not included in the article's Creative Commons licence and your intended use is not permitted by statutory regulation or exceeds the permitted use, you will need to obtain permission directly from the copyright holder. To view a copy of this licence, visit http://creativecommons.org/ licenses/by/4.0/. The Creative Commons Public Domain Dedication waiver (http://creativecommons.org/publicdomain/zero/1. 0/) applies to the data made available in this article, unless otherwise stated in a credit line to the data. 


\section{Introduction}

Migraine and cluster headache are primary headache disorders that share certain pathophysiological characteristics, but have a very different epidemiology and phenotype. Migraine prevalence is three times higher in women than in men [1]. Fluctuations in female sex hormones during the menstrual cycle and menopausal transition are associated with an increase in attack susceptibility, while pregnancy and postmenopausal status are associated with a decrease [2-4]. With migraine being a predominantly female disease, a limited number of studies has investigated sex hormones in men, but one small scale study showed a decreased testosterone/estradiol ratio in males with migraine [5].

As opposed to migraine, cluster headache was historically considered a male disease. Recent studies show that cluster headache occurs in women more often than previously assumed with a male to female ratio of $2: 1$ [6]. Onset of cluster headache before puberty is rare and cluster headache patients have been characterized as over-masculinized [7]. These clinical observations may suggest a role for androgens in cluster headache pathophysiology, but several small studies evaluating androgens, and testosterone in particular, have led to conflicting results.

Calcitonin gene-related peptide (CGRP) is known to be involved in the pathophysiology of migraine and cluster headache. Studies have indicated that (fluctuations in) sex hormones can modulate CGRP in the trigeminovascular system [8]. Sex hormones might thus play a role through CGRP. As relative androgen deficiency has been suggested in men with migraine, but may play a role in cluster headache as well, we aimed to compare symptoms of clinical androgen deficiency between male migraine and cluster headache patients and controls.

\section{Methods}

\section{Study design and participants}

We performed a cross-sectional questionnaire study among men with migraine, men with cluster headache and male controls without headache. Data were collected between October 2019 and April 2020. Participants were selected from the Leiden University Migraine Neuro Analysis (LUMINA) and the Leiden University Cluster headache neuro Analysis (LUCA) cohort [9, 10]. Men with migraine (episodic or chronic) or cluster headache (episodic or chronic) that fulfilled the International Classification of Headache Disorders (ICHD-3) criteria, and men without a primary or secondary headache disorder (apart from an occasional episodic tension-type headache), who gave written informed consent to be contacted in case of future research, were identified.

This study was approved by the medical ethics committee of Leiden University Medical Center. All subjects provided written informed consent.

\section{LUMINA and LUCA program}

Migraine patients, cluster headache patients and controls aged 18-80 years were recruited via nationwide public announcement, advertising in lay press and our research website. They were considered eligible after a two-step inclusion process using validated questionnaires. Additionally, patients attending our outpatient headache clinic were invited to participate. Migraine and cluster headache patients were first asked to fill out a validated web-based screening questionnaire with a sensitivity of 0.93 and specificity of 0.36 for migraine, and a sensitivity of 1.00 and specificity of 0.58 for cluster headache $[1,10]$. Patients who fulfilled the screening criteria for migraine or cluster headache, were sent a validated web-based extended migraine or cluster headache questionnaire, based on the International Classification of Headache Disorders criteria (previously ICHD-2, now ICHD-3 version) criteria [11]. The specificity of the extended migraine questionnaire was 0.95 and sensitivity was 0.45 [9]. This questionnaire is described in detail elsewhere [9]. The extended cluster headache questionnaire has a diagnostic specificity of 0.88 and sensitivity of $0.57[10,11]$.

We consider the cohort a well-defined web-based cohort. Four percent of migraine patients were included from our headache outpatient clinic and $87 \%$ of the participants were previously diagnosed with migraine by a physician. A clinically confirmed diagnosis of cluster headache by a physician was available for $94 \%$ of the LUCA population [10]. The LUCA questionnaire was primarily validated for the ICHD-2 criteria for cluster headache. However, all patients who fulfilled the ICHD2 criteria fulfilled the ICHD-3 criteria for cluster headache as well [12]. In addition to questions that were necessary to diagnose migraine and cluster headache accurately, the extended questionnaires also included items on demographic factors, aura and headache characteristics, acute and prophylactic headache medication use, and allodynia. Participants unable to use the web-based questionnaires due to lack of the needed internet skills were allowed to fill out the questionnaires on paper.

\section{Questionnaires}

Eligible subjects were invited to complete an online questionnaire with general questions about BMI, smoking and the use of (anti) androgen medication, and two validated questionnaires to assess clinical androgen status. In addition, patients provided migraine- or cluster headache specific information, whereas non-headache controls verified that they had not developed migraine or cluster headache since signing up as a control. Subjects using (anti) androgen medication and controls who had developed migraine or cluster headache were later excluded from all analyses. Reminders were sent out thrice to all subjects who had not yet participated. 
Two questionnaires were used to assess clinical androgen status: the Aging Males' Symptoms (AMS) scale, which consists of 17 items rated from 1 (none) to 5 (extremely severe) [13] and the quantitative Androgen Deficiency of Ageing Men (qADAM) questionnaire including 10 items scored on a Likert scale from 1 (absence of symptom) to 5 (maximal symptoms). The AMS scale is used to assess symptoms of aging across multiple countries and has an overall test-retest stability of 0.86 [14]. The qADAM questionnaire is adapted from the ADAM questionnaire which includes the same 10 items only dichotomized [15]. Both AMS and qADAM total scores are shown to be correlated with serum testosterone levels $[15,16]$. A higher AMS score corresponds with lower serum testosterone levels, while lower qADAM scores correspond with lower serum testosterone levels.

\section{Statistical analyses}

Primarily, generalized linear models were fitted to assess the mean difference in AMS and qADAM scores between men with migraine, cluster headache and non-headache controls. Since age, BMI and smoking are associated with alterations in the hypothalamic-pituitary-testicular axis and a consequent decline in testosterone, age, BMI, smoking and lifetime depression were included as covariates [17]. Age and BMI were treated as continuous variables, smoking as dichotomous variable and lifetime depression as categorical variable. Lifetime depression was defined as a HADS-D $\geq 8$ or CES-D $\geq 16$ or (past/present) depression diagnosed by a physician or (past/present) use of antidepressants for depression [18]. For some participants data on lifetime depression were missing, for whom it was set to unknown and analyzed as such.

As both the AMS and GADAM questionnaire contain multiple items associated with anxiety and depression, which are more prevalent in chronic headache disorders, we performed a secondary analysis determining the number of patients reporting to score below average $(\geq 4)$ on four selected items of the AMS scale regarding sexual symptoms (beard growth, morning erections, libido and sexual potency). In an earlier study these items were shown to differentiate aging symptoms from anxiety and depression [19]. Percentages of patients scoring below average were calculated for each group using descriptive statistics.

Additionally, exploratory analyses were performed for episodic versus chronic migraine and episodic versus chronic cluster headache. Chronic migraine was defined as an average of $\geq 15$ headache days per month during the past 3 months, from which $\geq 8$ days fulfil criteria for a migraine attack according to ICHD-3 [20]. Chronic cluster headache was defined as remission periods lasting < 3 months for at least 1 year according to ICHD-3 [20]. Furthermore, to rule out the influence of prophylactic medication, subgroup analyses were performed in migraine patients who did not use any prophylactic medication, and in episodic cluster headache patients out of bout.

Two-sided $p$-values $<0.05$ were considered statistically significant. Due to a clear distinction between primary and exploratory analyses, no corrections for multiple testing were made [21]. All analyses were performed in $\mathrm{R}$ version 3.6.1

\section{Results}

We sent an invitation to participate in this specific study to $n=853$ migraine patients, $n=694$ cluster headache patients, and $n=209$ controls without headache. Questionnaires were completed by $534 / 853$ (63\%) migraine patients, 437/694 (63\%) cluster headache patients and 152/209 (73\%) controls. Responders were slightly older

Table 1 Baseline characteristics for each group

\begin{tabular}{|c|c|c|c|}
\hline & Migraine & Cluster headache & Controls \\
\hline Number of patients, $n$ & 534 & 437 & 152 \\
\hline Age (years), mean \pm SD & $52.43 \pm 13.47$ & $53.23 \pm 12.92$ & $54.16 \pm 13.76$ \\
\hline $\mathrm{BMI}\left(\mathrm{kg} / \mathrm{m}^{2}\right)$, mean $\pm \mathrm{SD}$ & $25.43 \pm 3.54$ & $25.43 \pm 3.62$ & $25.12 \pm 3.12$ \\
\hline Smoking, n (\%) & $29(5.4)$ & $251(57.7)$ & $14(9.2)$ \\
\hline \multicolumn{4}{|l|}{ Lifetime depression, n (\%) } \\
\hline Yes & $196(36.7)$ & $160(36.6)$ & $15(9.9)$ \\
\hline No & $274(51.3)$ & $198(45.3)$ & $132(86.8)$ \\
\hline Unknown & $64(12.0)$ & $79(18.1)$ & $5(3.3)$ \\
\hline Chronic migraine, n (\%) & $82(15.4)$ & NA & NA \\
\hline Chronic CH, n (\%) & NA & $99(22.7)$ & NA \\
\hline
\end{tabular}

Abbreviations: $B M I$ body mass index, $S D$ standard deviation, $C H$ Cluster headache

Chronic migraine was defined based on the ICHD-3 criteria as $\geq 15$ headache days per month, from which $\geq 8$ migraine days. Chronic cluster headache was defined based on the ICHD-3 criteria as remission periods lasting $<3$ months for at least 1 year. Lifetime depression was defined as a HADS-D $\geq 8$ or CES-D $\geq 16$ or (past) depression diagnosed by a physician or (past) use of antidepressants for depression 


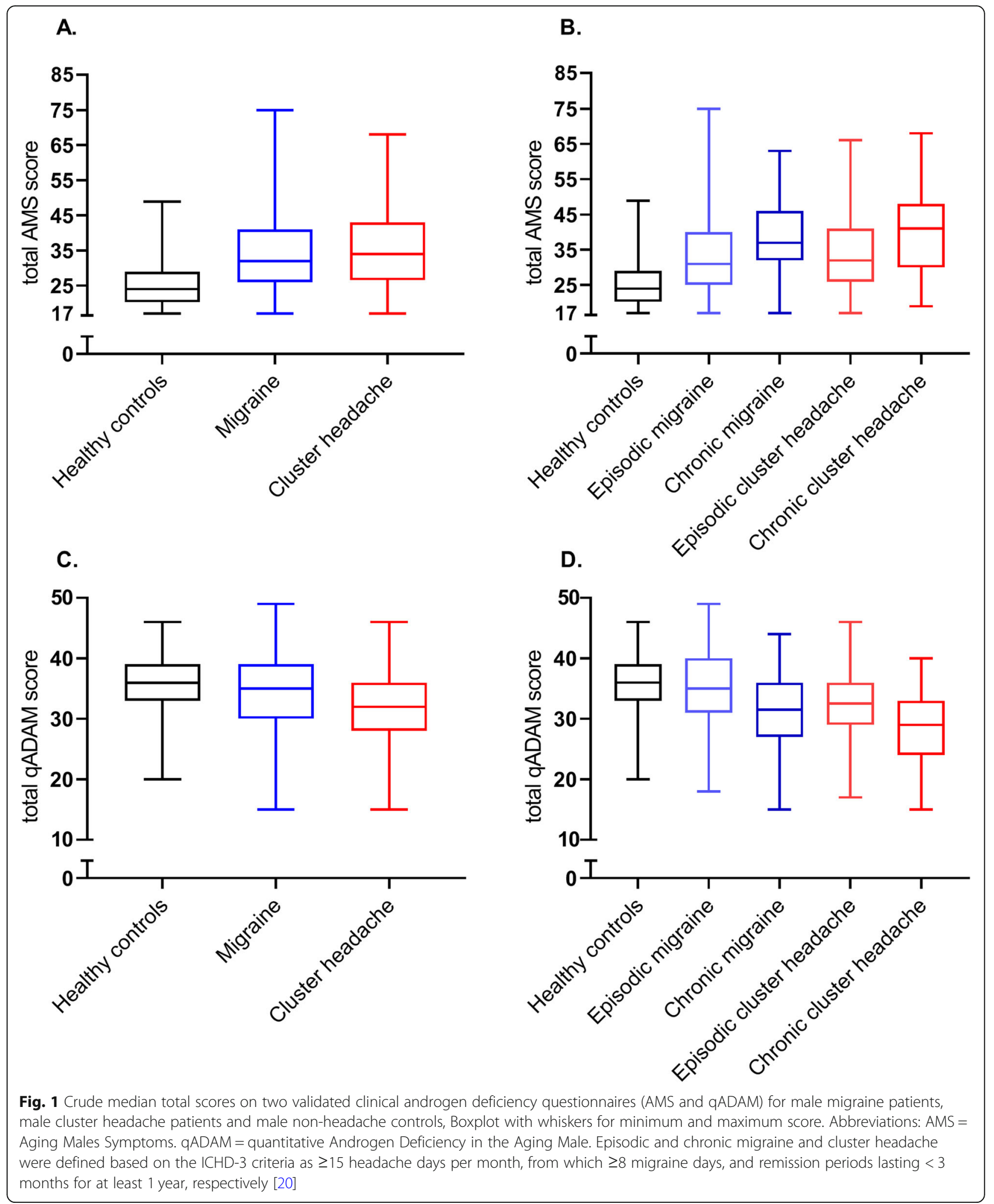

than non-responders and suffered less often from lifetime depression. No other differences were found between responders and non-responders for all three groups.
Patients with migraine, cluster headache and nonheadache controls were comparable regarding age and BMI. Patients with migraine and cluster headache were 
more likely to suffer from lifetime depression than healthy controls as was expected $(36.7 \%$ and $36.6 \%$ vs. 9.9\%). Patients with cluster headache smoked more often than healthy controls $(57.7 \%$ vs. $9.2 \%)$, while patients with migraine smoked less often than controls (5.4\% vs. 9.2\%). Baseline characteristics are shown in Table 1.

In the crude data, both migraine and cluster headache patients scored higher on the AMS (the higher the score, the more androgen deficiency symptoms) and lower on the qADAM scale (a lower score indicates more symptoms) than healthy controls, corresponding with more severe symptoms of clinical androgen deficiency. Patients with chronic migraine or cluster headache scored higher on the AMS and lower on the GADAM than episodic patients. Box plots showing the crude data can be found in Fig. 1.

\section{Primary analysis}

After adjusting for age, BMI, smoking and lifetime depression, differences were found in both AMS and qADAM scores between men with migraine, cluster headache and non-headache controls (see Tables 2 and 3). The mean AMS scores were higher in patients with migraine compared to controls (mean difference \pm SE: $5.44 \pm 0.90, p<0.001$ ), and cluster headache compared to controls $(5.62 \pm 0.99, p<0.001)$. Mean qADAM scores were lower in patients with migraine compared to controls $(-3.16 \pm 0.50, p<0.001)$, and cluster headache compared to controls $(-5.25 \pm 0.56, p<0.001)$.

\section{Exploratory analyses}

Subgroup analysis in migraine patients not using prophylactic medication $(n=242)$ compared to controls revealed similar results after correction for potential confounders for AMS total score (mean difference $\pm \mathrm{SE}$ :

Table 2 Results of generalized linear model with total AMS scale as dependent variable and headache type (control versus migraine versus cluster headache) as independent variable, corrected for age, BMI, smoking and lifetime depression. Control was set as the reference group

\begin{tabular}{llll}
\hline & $\boldsymbol{\beta}$ & SE & $\boldsymbol{p}$-value \\
\hline Intercept & 13.73 & 2.37 & $<0.001$ \\
Headache type & & & \\
$\quad$ Migraine & 5.44 & 0.90 & $<0.001$ \\
$\quad$ Cluster headache & 5.62 & 1.00 & $<0.001$ \\
Age & 0.02 & 0.02 & 0.28 \\
BMl & 0.37 & 0.08 & $<0.001$ \\
Smoking & 2.05 & 0.79 & 0.01 \\
Lifetime depression & & & \\
$\quad$ Yes & 9.67 & 0.65 & $<0.001$ \\
$\quad$ Unknown & 4.31 & 0.90 & $<0.001$ \\
\hline
\end{tabular}

Table 3 Results of generalized linear model with total qADAM score as dependent variable and headache type (control versus migraine versus cluster headache) as independent variable, corrected for age, BMI, smoking and lifetime depression. Control was set as the reference group

\begin{tabular}{llll}
\hline & $\boldsymbol{\beta}$ & SE & $\boldsymbol{p}$-value \\
\hline Intercept & 47.09 & 1.32 & $<0.001$ \\
Headache type & & & \\
$\quad$ Migraine & -3.16 & 0.50 & $<0.001$ \\
$\quad$ Cluster headache & -5.25 & 0.56 & $<0.001$ \\
Age & -0.06 & 0.01 & $<0.001$ \\
BMl & -0.18 & 0.05 & $<0.001$ \\
Smoking & -1.88 & 0.44 & $<0.001$ \\
Lifetime depression & & & \\
$\quad$ Yes & -4.53 & 0.36 & $<0.001$ \\
$\quad$ Unknown & -1.26 & 0.50 & 0.012 \\
\hline
\end{tabular}

$5.37 \pm 0.93, p<0.001$ ), and qADAM total score (mean difference \pm SE: $-2.59 \pm 0.57, p<0.001$ ). The number of monthly migraine days was weakly correlated with both AMS total score $(r=0.21,95 \%$ CI: 0.13 to 0.29 , $p<0.001)$, and qADAM total score $(r=-0.27,95 \% \mathrm{CI}$ : -0.34 to $-0.19, p<0.001$ ).

After correction for potential confounders, AMS total scores were higher in men with episodic cluster headache during a bout $(n=47)$ than out of bout $(n=291)$ (mean difference \pm SE: $4.76 \pm 1.58, p=0.007)$, while qADAM total scores were lower $(-3.13 \pm 0.84$, $p<0.001)$. Men with episodic cluster headache out of bout still scored higher on the AMS $(4.72 \pm 1.04$, $p<0.001)$ and lower on qADAM $(-4.62 \pm 0.58$, $p<0.001)$ than non-headache controls. No significant differences were found between men with episodic cluster headache during a bout and men with chronic cluster headache (AMS: $-0.22 \pm 1.65, \quad p=0.89$ / qADAM: $0.22 \pm 0.91, p=0.81$ ).

Additionally, migraine and cluster headache patients more often reported to score below average on each of the individual sexual items: decreased morning erections, libido and sexual potency, but not beard growth (see Fig. 2). Furthermore, more than twice as many patients with migraine (18.4\%) and cluster headache $(20.6 \%)$ reported to suffer from diminishment of at least one of these four sexual symptoms, compared with non-headache controls (7.2\%). Posthoc comparisons showed that statistically significant differences were present between men with migraine and non-headache controls (except for decreased libido), as well as between patients with cluster headache and non-headache controls (data not shown). Patients with chronic migraine $(15 \%$ of migraine participants) and chronic cluster headache $(23 \%$ of 

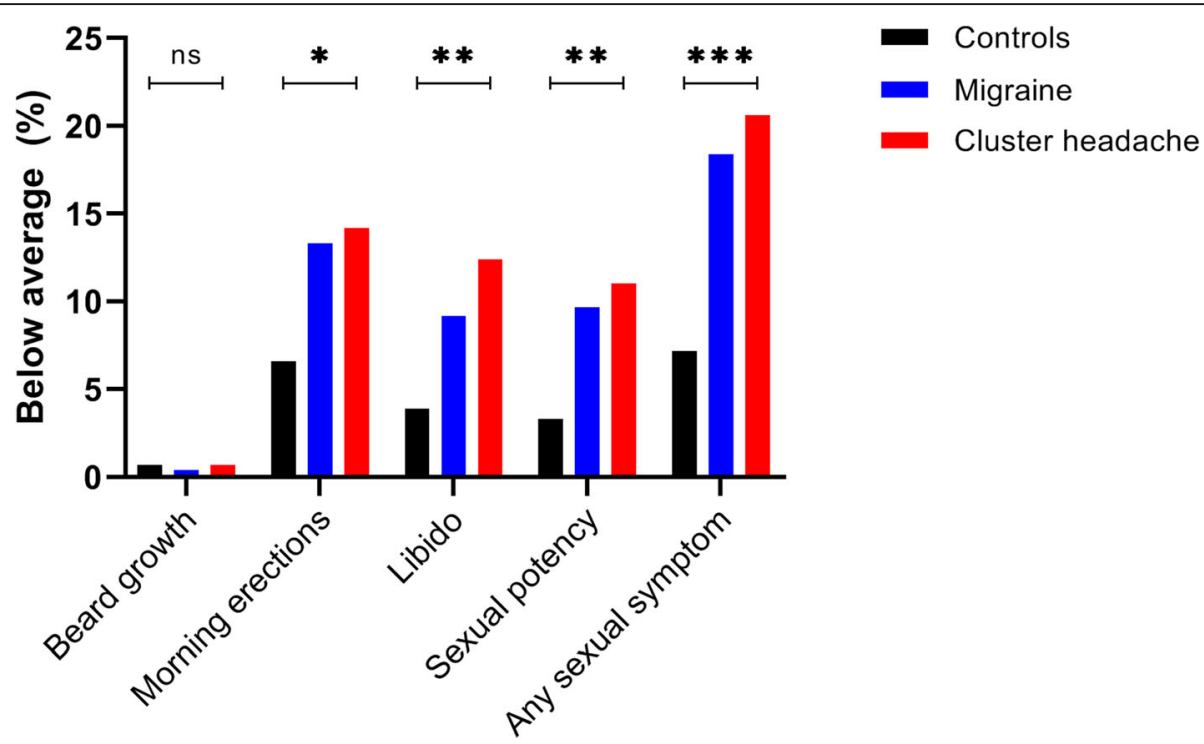
average on each of the individual items assessing sexual symptoms, and the percentage of participants suffering from diminishment of at least one of these four sexual symptoms. Abbreviations: NS = not significant; ${ }^{*}=p$-value $<0.05 ;^{* *}=p$-value $<0.01 ;{ }^{* * *}=p$-value $<0.001$

cluster headache participants) more often reported a diminishment of at least one sexual symptom (see Fig. 3 and Table 4).

\section{Discussion}

This cross-sectional study shows that men with migraine and cluster headache more often suffer from symptoms consistent with (relative) clinical androgen deficiency than males without a primary headache disorder. In addition, our study shows that these symptoms are more pronounced in men with chronic migraine, i.e. a high attack frequency, and chronic cluster headache, i.e. short or no remission periods.

Androgen deficiency is associated with a wide range of symptoms, ranging from mood disturbances to sexual symptoms such as decreased libido and number of morning erections. Our study shows that patients with migraine and cluster headache suffer more frequently and more severely from these symptoms than men without headache. Whether this is the result of hormonal imbalances or an epiphenomenon reflecting chronic disease remains to be elucidated.

Only limited evidence exists on hormone levels in men with migraine and cluster headache. A recent study in male migraine patients showed symptoms of a relative androgen deficiency with a higher estradiol/testosterone ratio compared to controls, which was attributed to higher estradiol levels [5]. No significant differences were found in free testosterone levels. In contrast, one very small-scale study described low testosterone levels in men with chronic migraine compared to age-matched normative values [22]. Similarly, in contrast to the concept of 'over-masculinization', several older studies suggest a lower testosterone level in men with cluster headache [23-27]. These presumed differences in estra$\mathrm{diol} /$ testosterone ratios may be responsible for the symptomatology described in the present study, which would strengthen the hypothesis of hormonal imbalances in men with migraine and cluster headache.

As scientific literature on hormone levels in men with primary headache disorders is scarce, it remains unknown whether hormone imbalances contribute to migraine or cluster headache pathophysiology, or that these observations are the result of an (even possibly unrelated) epiphenomenon. An interesting study in male-to-female transsexuals who use antiandrogens and estrogens reported migraine rates similar to genetic females, adding to the notion that sex hormones may contribute to migraine prevalence and possibly migraine pathophysiology [28]. Another diseasemodifying effect of androgens was reported in two small scale trials in women in whom improvement in migraine was reported after either implantation of a testosterone pellet or the use of danazol, a steroid hormone $[29,30]$. Positive effects of testosterone replacement therapy in a very small group of cluster headache patients was reported as well [31].

A major limitation of the current study is that we used questionnaires as an indirect measure of hormone levels. Therefore, it may be argued that the symptomatology described may not be very specific, and might also be attributed to long-term effects of a chronic disease status, instead of (relative) androgen deficiency. Previous studies have shown that both AMS and GADAM 


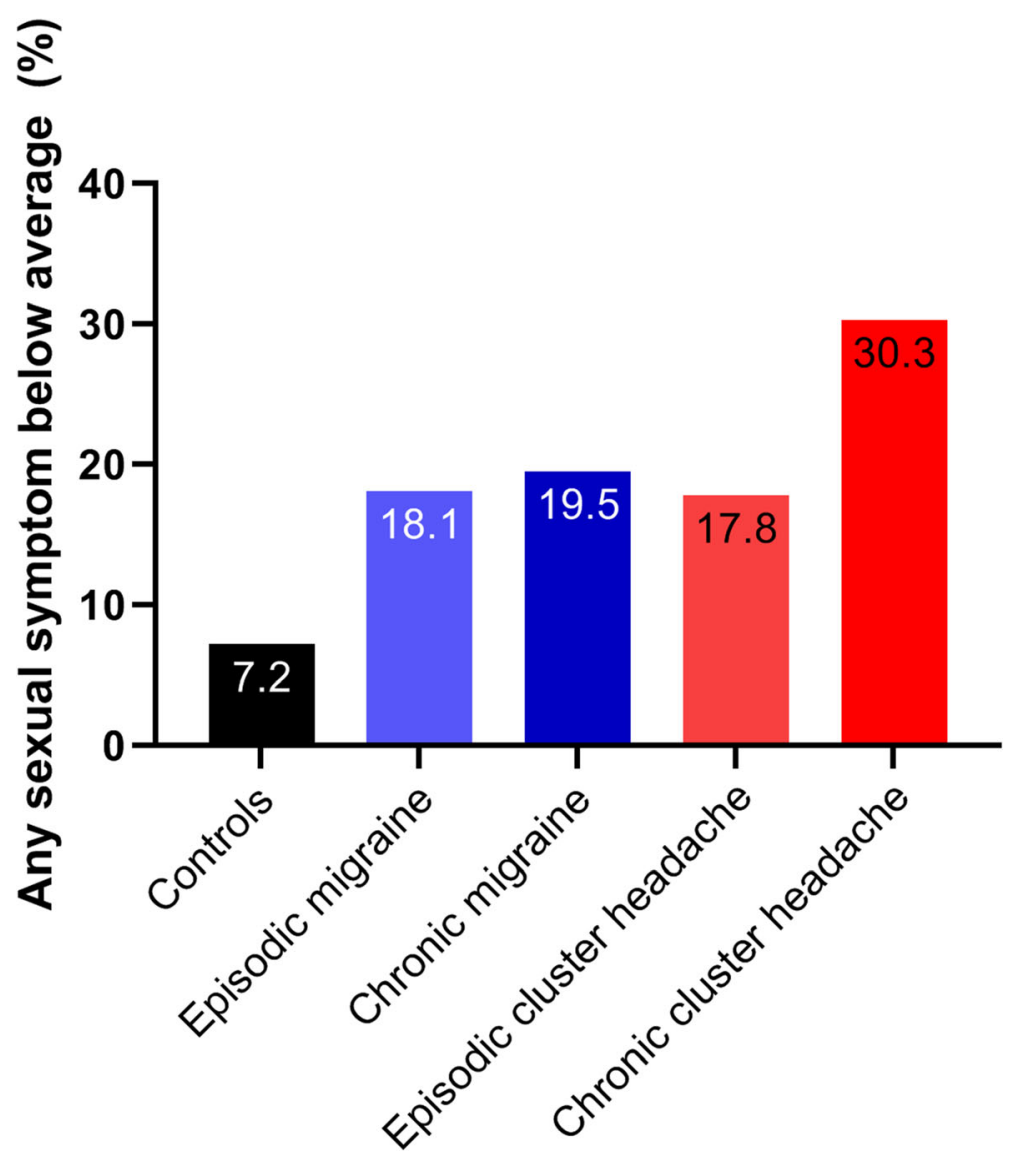

Fig. 3 The percentage of male migraine patients, male cluster headache patients and male non-headache controls reporting to score below average on at least one of four specific sexual symptoms; beard growth, morning erections, libido and sexual potency. Abbreviations: AMS = Aging Males Symptoms. qADAM = quantitative Androgen Deficiency in the Aging Male. Episodic and chronic migraine and cluster headache were defined based on the ICHD-3 criteria as $\geq 15$ headache days per month, from which $\geq 8$ migraine days, and remission periods lasting $<3$ months for at least 1 year, respectively [20]

Table 4 The percentage of male migraine patients, male cluster headache patients and male non-headache controls reporting to score below average on each of the individual items assessing sexual symptoms and the percentage of participants suffering from diminishment of at least one of these four sexual symptoms

\begin{tabular}{lllll}
\hline & Controls & Migraine & Cluster headache & $\boldsymbol{p}$-value \\
\hline $\mathrm{n}$ & 152 & 534 & 437 & $3(0.7)$ \\
Beard growth & $1(0.7)$ & $2(0.4)$ & $62(14.2)$ & 0.782 \\
Morning erections & $10(6.6)$ & $71(13.3)$ & $54(12.4)$ & 0.046 \\
Libido & $6(3.9)$ & $49(9.2)$ & $52(11.9)$ & 0.009 \\
Sexual potency & $5(3.3)$ & $52(9.7)$ & $90(20.6)$ & 0.008 \\
Any sexual symptom & $11(7.2)$ & $98(18.4)$ & $<0.001$ \\
\hline
\end{tabular}


questionnaires display a (moderate) correlation with serum testosterone levels $[14,15]$. However, these questionnaires were developed and validated in the aging male and have not been validated in other patient groups such as headache patients. Both questionnaires contain multiple items that are known to be associated with chronic headache disorders such as mood disturbances, anxiety and sleeping problems. We corrected for these confounding factors by including them as covariates in our analyses [32-37]. After correction, differences between headache patients and controls remained present. In addition we performed a secondary analysis, incorporating more robust items that were previously shown to differentiate androgen deficiency symptoms from anxiety and depression [19, 38-42]. This secondary analysis showed that patients with migraine and cluster headache more often report impaired sexual symptomatology, which more specifically points in the direction of androgen deficiency.

Furthermore, the symptomatology described in this study could partly be explained by side effects of prophylactic medication. However, a subgroup analysis in migraine patients who did not use any prophylactic drugs and episodic cluster headache patients out of bout, revealed similar results, making a (large) confounding effect unlikely. We therefore assume that the use of prophylactic drugs has a negligible influence on our final results.

In conclusion, our study shows that men with migraine and cluster headache more frequently report symptomatology consistent with androgen deficiency than males without a primary headache disorder. We hypothesize that these differences may be the result of a (relative) androgen deficiency, which can be attributed to either higher estradiol or lower testosterone levels. However, suffering from a chronic (headache) disorder might be implicated as well. Regardless of the cause, it is important to recognize this symptomatology in patients since this may negatively impact quality of life. We advise physicians to proactively assess mood and sexual symptoms in patients with migraine and cluster headache.

\section{Abbreviations \\ AMS: Aging Males Symptoms; qADAM: Quantitative Androgen Deficiency in the Aging Male}

\section{Acknowledgements}

Not applicable.

\section{Authors' contributions}

I.E. Verhagen: Design and conceptualized study; Major role in the acquisition of data; analyzed the data; Interpreted the data; drafted the manuscript for intellectual content. R.B. Brandt: Design and conceptualized study; Major role in the acquisition of data; analyzed the data; Interpreted the data; revised the manuscript for intellectual content. C.M.A. Kruitbosch: Design and conceptualized study; Major role in the acquisition of data; Interpreted the data; revised the manuscript for intellectual content. A. MaassenVanDenBrink:
Design and conceptualized study; Major role in the acquisition of data; Interpreted the data; revised the manuscript for intellectual content. R. Fronczek: Design and conceptualized study; Major role in the acquisition of data; Interpreted the data; revised the manuscript for intellectual content. G.M. Terwindt: Design and conceptualized study; Major role in the acquisition of data; Interpreted the data; revised the manuscript for intellectual content. I.E. Verhagen and G.M. Terwindt read and approved the final manuscript. The author(s) read and approved the final manuscript.

\section{Funding}

None.

Availability of data and materials

The datasets used and/or analysed during the current study are available from the corresponding author on reasonable request.

\section{Declarations}

Ethics approval and consent to participate

This study was approved by the medical ethics committee of Leiden University Medical Center. All subjects provided written informed consent.

Consent for publication

Not applicable.

\section{Competing interests}

I.E. Verhagen has received research grants from ZonMw and the Dutch Brain Foundation. R.B. Brandt has received support for conference visits from Allergan. C.M.A. Kruitbosch reports no disclosures. A. MaassenVanDenBrink reports consultancy or industry support from Novartis, Lilly and Teva, and independent support from the Dutch Research Council (VICl grant 09150181910040), The Netherlands Organisation for Health Research and Development (ZonMw) and the Dutch Heart \& Brain Foundations. R. Fronczek has received consultancy support from Novartis, Lilly, Teva, Allergan, and independent support from the Dutch Brain Foundation and Innovation Fund Dutch Healthcare Providers. G.M. Terwindt reports consultancy support from Novartis, Allergan, Lilly, and Teva, and independent support from Dutch Organization for Scientific Research, the Dutch Heart \& Brain Foundations, IRRF and Dioraphte.

\section{Author details}

'Department of Neurology, Leiden University Medical Center, P.O. 9600, 2300, WB, Leiden, the Netherlands. 'Department of Internal Medicine, Erasmus University Medical Center, Rotterdam, The Netherlands.

Received: 15 July 2021 Accepted: 24 September 2021

Published online: 19 October 2021

\section{References}

1. Launer LJ, Terwindt GM, Ferrari MD (1999) The prevalence and characteristics of migraine in a population-based cohort: the GEM study. Neurology 53:537-542

2. Kvisvik EV, Stovner L, Helde G, Bovim G, Linde M (2011) Headache and migraine during pregnancy and puerperium: the MIGRA-study. J Headache Pain 12:443-451

3. Neri I, Granella F, Nappi R, Manzoni GC, Facchinetti F, Genazzani AR (1993) Characteristics of headache at menopause: a clinico-epidemiologic study. Maturitas 17:31-37

4. Nappi RE, Albani F, Sances G, Terreno E, Brambilla E, Polatti F (2011) Headaches during pregnancy. Curr Pain Headache Rep 15:289-294

5. van Oosterhout WPJ, Schoonman GG, van Zwet EW et al (2018) Female sex hormones in men with migraine. Neurology 91:e374-e381

6. Frederiksen $\mathrm{HH}$, Lund NL, Barloese MC, Petersen AS, Jensen RH. Diagnostic delay of cluster headache: A cohort study from the Danish Cluster Headache Survey. Cephalalgia. 2020;40(1):49-56. https://doi.org/10.1177/ 0333102419863030 . Epub 2019 Jul 10

7. Manzoni GC, Micieli G, Granella F, Tassorelli C, Zanferrari C, Cavallini A (1991) Cluster headache course over ten years in 189 patients. Cephalalgia 11:169-174

8. Labastida-Ramírez A, Rubio-Beltrán E, Villalón CM, MaassenVanDenBrink A (2019) Gender aspects of CGRP in migraine. Cephalalgia 39:435-444 
9. van Oosterhout WP, Weller CM, Stam AH et al (2011) Validation of the webbased LUMINA questionnaire for recruiting large cohorts of migraineurs. Cephalalgia 31:1359-1367

10. Wilbrink LA, Weller CM, Cheung C et al (2013) Stepwise web-based questionnaires for diagnosing cluster headache: LUCA and QATCH. Cephalalgia 33:924-931

11. Headache Classification Committee of the International Headache Society (2018) The international classification of headache disorders, 3rd edition. Cephalalgia 38:1-211

12. De Coo I, Wilbrink L, Haan J, Ferrari M, Terwindt G (2016) Evaluation of the new ICHD-3 beta cluster headache criteria. Cephalalgia 36:547-551

13. Heinemann LA (2005) Aging Males' symptoms scale: a standardized instrument for the practice. J Endocrinol Investig 28:34-38

14. Daig I, Heinemann LA, Kim S et al (2003) The aging Males' symptoms (AMS) scale: review of its methodological characteristics. Health Qual Life Outcomes 1:77

15. Mohamed O, Freundlich RE, Dakik HK et al (2010) The quantitative ADAM questionnaire: a new tool in quantifying the severity of hypogonadism. Int $J$ Impot Res 22:20-24

16. Kratzik CW, Reiter WJ, Riedl AM et al (2004) Hormone profiles, body mass index and aging male symptoms: results of the Androx Vienna municipality study. Aging Male 7:188-196

17. Wu FC, Tajar A, Pye SR et al (2008) Hypothalamic-pituitary-testicular axis disruptions in older men are differentially linked to age and modifiable risk factors: the European male aging study. J Clin Endocrinol Metab 93:27372745

18. Louter MA, Pelzer N, de Boer I et al (2016) Prevalence of lifetime depression in a large hemiplegic migraine cohort. Neurology 87:2370-2374

19. Lee CP, Jiang JR, Chen $Y$ et al (2013) The "aging Males' symptoms" (AMS) scale assesses depression and anxiety. Aging Male 16:97-101

20. Classification Committee of the International Headache Society (2018) Headache Classification Committee of the International Headache Society (IHS) The International Classification of Headache Disorders, 3rd edition. Cephalalgia 38:1-211

21. Groenwold RHH, Goeman JJ, Cessie SL, Dekkers OM (2021) Multiple testing: when is many too much? Eur J Endocrinol 184:E11-e14

22. Shields LBE, Seifert T, Shelton BJ, Plato BM (2019) Testosterone levels in men with chronic migraine. Neurol Int 11:8079

23. Facchinetti F, Nappi G, Cicoli C et al (1986) Reduced testosterone levels in cluster headache: a stress-related phenomenon? Cephalalgia 6:29-34

24. Romiti A, Martelletti P, Gallo MF, Giacovazzo M (1983) Low plasma testosterone levels in cluster headache*. Cephalalgia 3:41-44

25. Klimek A (1982) Plasma testosterone levels in patients with cluster. Headache. 22:162-164

26. Nelson RF (1978) Testosterone levels in cluster and non-cluster Migrainous headache patients. Headache 18:265-267

27. Murialdo G, Fanciullacci M, Nicolodi M, Filippi U, De Palma D, Sicuteri F, Polleri A. Cluster headache in the male: sex steroid pattern and gonadotropic response to luteinizing hormone releasing hormone. Cephalalgia. 1989;9(2):91-8. https://doi.org/10.1046/j.1468-2982.1989.0902091. $x$.

28. Pringsheim T, Gooren $L$ (2004) Migraine prevalence in male to female transsexuals on hormone therapy. Neurology 63:593-594

29. Glaser R, Dimitrakakis C, Trimble N, Martin V (2012) Testosterone pellet implants and migraine headaches: a pilot study. Maturitas 71:385-388

30. Lichten EM, Bennett RS, Whitty AJ, Daoud Y (1991) Efficacy of danazol in the control of hormonal migraine. J Reprod Med 36:419-424

31. Stillman MJ (2006) Testosterone replacement therapy for treatment refractory cluster headache. Headache 46:925-933

32. Allen NE, Appleby PN, Davey GK, Key TJ (2002) Lifestyle and nutritional determinants of bioavailable androgens and related hormones in British men. Cancer Causes Control 13:353-363

33. English KM, Pugh PJ, Parry H, Scutt NE, Channer KS, Jones TH (2001) Effect of cigarette smoking on levels of bioavailable testosterone in healthy men. Clin Sci (Lond) 100:661-665

34. Gapstur SM, Gann PH, Kopp P, Colangelo L, Longcope C, Liu K (2002) Serum androgen concentrations in young men: a longitudinal analysis of associations with age, obesity, and race. The CARDIA male hormone study. Cancer Epidemiol Biomark Prev 11:1041-1047

35. Harman D (2001) Aging: overview. Ann N Y Acad Sci 928:1-21
36. Wang W, Yang X, Liang J et al (2013) Cigarette smoking has a positive and independent effect on testosterone levels. Hormones (Athens) 12:567-577

37. Wildman RP, Wang D, Fernandez I et al (2013) Associations of testosterone and sex hormone binding globulin with adipose tissue hormones in midlife women. Obesity (Silver Spring) 21:629-636

38. Kim BS, Chung PW, Kim BK et al (2020) The impact of remission and coexisting migraine on anxiety and depression in cluster headache. J Headache Pain 21:58

39. Louter MA, Wilbrink LA, Haan J et al (2016) Cluster headache and depression. Neurology 87:1899-1906

40. Robbins MS, Bronheim R, Lipton RB et al (2012) Depression and anxiety in episodic and chronic cluster headache: a pilot study. Headache 52:600-611

41. Dindo LN, Recober A, Haddad R, Calarge CA (2017) Comorbidity of migraine, major depressive disorder, and generalized anxiety disorder in adolescents and young adults. Int J Behav Med 24:528-534

42. Rist PM, Schurks M, Buring JE, Kurth T (2013) Migraine, headache, and the risk of depression: prospective cohort study. Cephalalgia 33:1017-1025

\section{Publisher's Note}

Springer Nature remains neutral with regard to jurisdictional claims in published maps and institutional affiliations.

\section{Ready to submit your research? Choose BMC and benefit from:}

- fast, convenient online submission

- thorough peer review by experienced researchers in your field

- rapid publication on acceptance

- support for research data, including large and complex data types

- gold Open Access which fosters wider collaboration and increased citations

- maximum visibility for your research: over $100 \mathrm{M}$ website views per year

At BMC, research is always in progress.

Learn more biomedcentral.com/submissions 